\title{
Phenotypic and Molecular Characterization of Antibiotic resistance of Isolated Salmonella Strains from Chickens in Côte D'ivoire
}

\author{
Bonny Aya Carole* and Karou Tago Germain
}

\begin{abstract}
Laboratory of Biotechnology, Agriculture and Development of Biological Resources, of the Biosciences Training and Research Unit, Félix Houphouët-Boigny University, Abidjan, Côte d'Ivoire, 22 BP 582 Abidjan 22.
\end{abstract}

*Corresponding author

Keywords

Antibiotic-resistant Salmonella,

chickens, food

safety, Côte d'Ivoire

Article Info

Accepted:

12 June 2021

Available Online:

10 July 2021
Poultry consumption in Côte d'Ivoire is booming, however it is the main reservoir of antibiotic resistant strains of Salmonella. The objective of this work is to assess the level of resistance to Salmonella antibiotics isolated from chickens. Salmonella strains (104) isolated from 51 batches of raw chicken gedisers were subjected to phenotypic and molecular characterization. Derby (18.9\%), Budapest (17\%), Essen and Kentucky (11.3\%) represent the predominant serotypes. The antibiogram carried out showed resistance: high to cotrimoxazole $(93.37 \%)$ and to tetracycline $(73.08 \%)$; relatively moderate for ticarcillin $(46.15 \%)$ and ciprofloxacin $(28.85 \%)$ and lower for cefotaxime (0.96\%). The resistance genes tet (A), bla CTX-M-1, bla CTX-Mconsensus, sul 1, qnr (A, B and S), sought by molecular tests (PCR and sequencing) revealed the presence of genes tet (A) (40\%), sul 1 (40\%), bla CTX-M-1 $(65 \%)$ and the presumption of a diversity of bla genes including: CTX-M-2, $5,-44,-59,-92,-97$, OXY and NDM-1. Therefore, monitoring the use of antibiotics in poultry farming remains an essential precaution to guarantee the safety of food intended for human consumption.

\section{Introduction}

Foodborne illnesses are a major cause of morbidity and mortality across the world. The World Health Organization (WHO) estimates that 2 million people die each year from infectious diarrhea (Anonymous 1, 2006). Of these, salmonellosis is a real problem in all parts of the world. Indeed, they have a considerable importance in the veterinary and medical fields, as much by the economic losses linked to the reduction in production, as by the high incidence of collective food poisoning, in a current context where absolute sanitary safety is required by the consumer. Salmonellosis is one of the main causes of 
foodborne gastroenteritis in humans (Anonymous 2, 2002). They cause symptoms of a wide range of severity, from mild abdominal pain and varying degrees of enteritis, to sepsis and in extreme cases, death. Salmonella enterica, through its ubiquitous serotypes, represents the main pathogenic agent in the contamination of agro-food products intended for human consumption (Fablet et al., 2003).

Salmonella infection is also very commonly associated with the consumption of meat and meat products, especially those made from poultry. In fact, poultry play a major role as vectors of transmission in human cases of salmonellosis (Anonymous 2, 2002). Otherwise, the consumption of poultry meat has grown considerably on all continents with an increase in volumes sold worldwide, by $10 \%$ per year (Prin et al., 2001). However, in Côte d'Ivoire, farming and slaughtering practices are lagging far behind in industrialized countries, not only with regard to the productivity of poultry workshops, but also and above all with regard to public health.

The increase and accumulation of resistance to antibiotics by Salmonella is another aspect of the public health problem, because it is accepted that some of the multidrug-resistant strains found in humans are of animal origin and have acquired their genes from resistance in farms before being transmitted to humans through food (Ungemach et al., 2006).

In fact, the continued use of antibiotics has led to the selection of resistant germs (Anonymous 3, 2009) with the consequences of an increase in infections in chickens, an increase in the mortality rate and a reduction in the productivity of an animal. go; and the possible transfer of this resistance from chicken to humans on the other hand (Jianhua et al., 2002; Bourgeois et al., 2003; Moubareck et al., 2003).

\section{Materials and Methods}

The animal material consists of raw chicken gizzards taken from poultry slaughtering sites in the District of Abidjan. Reference bacterial strains (Salmonella ATCC 14028 and IPCI 8297) were used as a positive control for carrying out the various biochemical tests, as well as to validate the tests for studying resistance to antibiotics. Six strains of Escherichia coli (E. coli PSL 18X61367- E. coli Y10278- E. coli X92506- E. coli DJ2115- E. Coli J53 PMG252 and E. coli 57) served as positive control for detection respectively tet(A), bla CTX-M consensus, bla CTX-M-1 (group 1), sul 1, qnr (A) and $q n r$ (S) genes. Klebsiella pneumoniae B1 served as a positive control for the $q n r$ (B) gene. Six pairs of specific primers and a pair of universal primers (Eurogentec, France) were used for the search for antibiotic resistance genes (Table 1).

\section{Sampling and microbiological analysis for the detection of Salmonella}

Batches (66) of raw gizzards were taken from slaughtering sites in 11 communes of the district of Abidjan (Abobo, Adjamé, Anyama, Attécoubé, Bingerville, Cocody, Koumassi, Marcory, Port-Bouët, Treichville and Yopougon), from April to September 2012.

The microbiological analysis of the different batches of raw gizzards was carried out according to standard NF EN ISO 6579 (ISO6579, 2002) comprising 4 stages: preenrichment, enrichment, isolation and biochemical identification.

\section{Serotyping of isolated Salmonella strains}

The serotyping of Salmonella was carried out according to the method described by Kauffmann and White (1934), consisting in successively detecting somatic (Ag O), 
flagellar $\mathrm{H}(\mathrm{Ag} H)$ or capsule $\mathrm{Ag}(\mathrm{Vi})$ antigens, by agglutination on slide using antigenic sera.

\section{Determination of antibiotic resistance}

The antibiogram carried out on all the strains isolated was carried out by diffusion in agar medium according to the CLSI standard (Clinical Laboratory Standard Institute) on Müller-Hinton agar (CLSI, 2005). Antibiotic

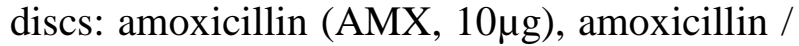
clavulanic acid combination (AMC, 10 / $20 \mu \mathrm{g}$ ), ticarcillin (TIC, $75 \mu \mathrm{g}$ ), cefalotin (CF,

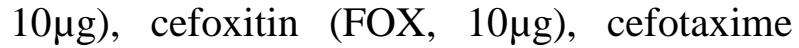

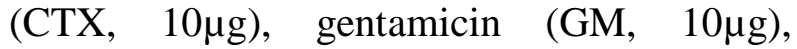
nalidixic acid (Nal, 10 $\mu$ g), ciprofloxacin (Cip, $10 \mu \mathrm{g})$, cotrimoxazole (SXT, $10 / 20 \mu \mathrm{g}$ ),

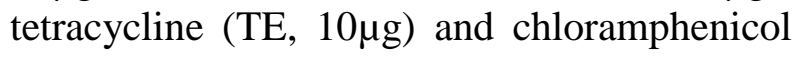
$(\mathrm{C}, 10 \mu \mathrm{g})$, were tested.

\section{Detection and amplification of resistance genes by PCR}

The detection of genetic carriers of antibiotic resistance was carried out by the polymerase chain reaction (PCR) technique on 20 strains of Salmonella exhibiting a profile of multidrug resistance. The search for certain resistance markers including: the bla CTX-M-1 (group 1) and bla CTX-Mconsensus genes encoding resistance to $\beta$-lactams (ticarcillin, cefotaxime); the tet(A) gene encoding resistance to cyclins (tetracycline), the sul1 gene encoding resistance to sulfonamides (cotrimoxazole) and the $q n r$ genes (A, B and S) encoding resistance to fluoroquinolones (ciprofloxacin), a been carried out. The genetic material (plasmid DNA) was extracted according to the method described by Rozilla et al., (2007), then amplified using primers (Table 1). The amplification products were subjected to electrophoresis on 1\% agarose gel (Eurobio, France) and the target genes were revealed under UV. The gene amplification reaction was carried out using a thermocycler
(Applied Biosystems Gene Amp PCR 9700), in a reaction mixture of $50 \mu \mathrm{L}$. The gene amplification program comprises an initial denaturation of $5 \mathrm{~min}$ at $94^{\circ} \mathrm{C}$, followed by 40 cycles of PCR, each of which consists of a denaturation step of $30 \mathrm{~s}$ at $94^{\circ} \mathrm{C}$; a hybridization step for $1 \mathrm{~min}$ at $55^{\circ} \mathrm{C}$. for the pair of primers bla CTX-Mconsensus; at $60^{\circ}$ C. for the pairs of primers bla CTX-M-1 (group 1), tet (A) and $q n r(\mathrm{~A}, \mathrm{~B}, \mathrm{~S})$; at $69^{\circ} \mathrm{C}$ for the initiator pair sul 1; in a one-minute elongation step at $72^{\circ} \mathrm{C}$. At the end of the 40 cycles, a final elongation of 10 minute at $72^{\circ}$ $\mathrm{C}$, completes the amplication reaction.

\section{Sequencing of amplified genes}

The DNA amplicons obtained by PCR from the degenerate primer bla CTX-Mconsensus are sequenced at GATC Biotech (Germany). The nucleotide sequences obtained are identified using the NCBI (National Center for Biotechnology Information) database, available on the website www.blast.ncbi.nlm.gov/Blast.cgi.

\section{Results and Discussion}

Microbiological analysis revealed the microbiological quality of raw chicken gizzards. Thus, out of the 66 batches of gizzards analyzed, 51 batches were contaminated by Salmonella, ie a percentage of contaminated batches of $77.27 \%$. From these contaminated batches, 104 strains of Salmonella were isolated. Of all the serotyped strains, 15 serotypes including 11 agglutinating with serum OMA and 4 with serum OMB could be determined. The serotypes derived from strains agglutinating with the OMA serum are: Derby (18.9\%), Budapest (17\%), Essen (11.3\%), Agona (7.5\%), Chester (3.8\%), Schwarzen ground (3.8\%), Ruiru (3.8\%), Fortune (1.9\%), Elisabethville (1.9\%), Aoto (1.9\%), and Santiago (1.9\%). Those derived from strains 
agglutinating with OMB serum are: Kentucky (11.3\%), Hadar (9.4\%), Bargny (1.9\%), and Poeselderf (1.9\%).

The study of antibiotic resistance of Salmonella strains, carried out on all strains showed resistance to $\beta$-lactams (ticarcillin $(46.15 \%)$ ), sulfonamides (cotrimoxazole $(93.27 \%)$ ), quinolones (nalidixic acid $(35.76 \%)$ and ciprofloxacin $(28.85 \%)$ ) and cyclins (tetracyclines (73.08\%)). Cyclins and sulfonamides remain the least active antibiotic families against isolated Salmonella strains. The antibiogram also revealed resistance profiles ranging from mono resistance to multiple resistance $(3,4,5,6,8,9$ and 11 molecules). The serotypes involved in multidrug resistance are: Agona (17.39\%), Derby (8.69\%), Hadar (4.34\%), Budapest (21.73\%), Ruiru (8.69\%), Essen (17.39\%), Kentucky (17.39\%) and Chester (4.34\%) (Table 2).

Electrophoresis of PCR products revealed the presence of markers implicated in antibiotic resistance of Salmonella strains isolated from raw chicken gizzards (Figure 1). None of the targeted Salmonella strains possess the fluoroquinolone resistance genes (qnr (A, B, S)).

The sequencing carried out on the amplicons of the degenerate primer bla CTXMconsensus at the level of two strains (Salmonella Kentucky and Salmonella O: 3,10 ), revealed similarities of 96 to $100 \%$ with fragments of nucleotide sequences encoding bla CTX-M-2, -5, -44, -59, -92, 97 and -131 enzymes; bla NDM-1 and bla OXY (Table 3). Also sequencing reveals the presence of mobile genetic elements such as ISEcp1 type insertion sequences and ISCRI.

The isolation of the Salmonella strains from the different batches of gizzards analyzed revealed a rate of contaminated batches of
$77.27 \%$. The presence of these strains in the chicken lays bare the process of treating slaughtered chickens. Indeed, this process constitutes an important means of diffusion of microorganisms such as Salmonella. Salmonella strains are isolated from viscera (Gaedirelwe and Sebunya, 2008; Traoré, 2003), and gizzards indirectly contaminated by the intestinal contents of chicken (Chaiba et al., 2008; Karou et al., 2013).

The serotyping carried out on all the strains isolated revealed 15 serotypes. Derby (18.9\%), Budapest (17\%), Essen (11.3\%) and Kentucky $(11.3 \%)$ represent the most dominant serotypes. Indeed, since 2000, the Derby and Kentucky serotypes have been the main Salmonella serotypes most widely distributed in France and Belgium (Weill and Le Hello, 2011; Bertrand et al., 2010). Also, some studies have shown the existence of these serotypes in Salmonella strains isolated from various sources including poultry (Tao et al., 2014; Karraouan et al., 2010; Turki et al., 2011). The Kentucky serotype, in particular, remains an emerging serotype, associated with strains highly resistant to critical molecules such as fluoroquinolones (ciprofloxacin), recommended in cases of severe infections,

Analysis of the resistance profile of multiresistant strains of Salmonella to antibiotics revealed 4 levels of multiple resistance, involving several different families of antibiotics. $\quad \beta$-lactams, cyclins, sulfonamides and quinolones are the most affected in these multiple resistances. The appearance of these combinations involving these different families would be the direct consequence of their overuse in the Ivorian poultry sector (Ouattara et al., 2013). Indeed, despite the WHO recommendations on the use of antibiotics in farms, molecules similar to those used in clinics are still used in some countries and are undoubtedly at the origin of the appearance of cross-resistance. 
Table.1 Primer pairs of antibiotic resistance genes used during the study.

\begin{tabular}{|c|c|c|c|c|}
\hline Target genes & Nucleotide sequences (5' & Function & $\begin{array}{l}\text { Amplicon } \\
\text { (bp) }\end{array}$ & References \\
\hline bla CTX-Mc & $\begin{array}{l}\text { F: ATGTGCAGYACCAGTAARGTKATGGC } \\
\text { R:TGGGTRAARTARGTSACCAGAAYCAGCGG }\end{array}$ & Search for blaCTX-M genes & 593 & Kiiru et al. (2012) \\
\hline bla CTX-M1 & $\begin{array}{l}\text { F: GACGATGTCACTGGCTGAGC } \\
\text { R: AGCCGCCGACGCTAATACA }\end{array}$ & Search for bla CTX-M1 genes & 499 & Kiiru et al. (2012) \\
\hline tet (A) & $\begin{array}{l}\text { F: GCTACATCCTGCTTGCCTTC } \\
\text { R: CATAGATCGCCGTGAAGAGG }\end{array}$ & Search for tet genes (A) & 210 & Ng et al. (2001) \\
\hline sul 1 & $\begin{array}{l}\text { F: CTTCGATGAGAGCCGGCGGC } \\
\text { R: GCAAGGCGGAAACCCGCGCC }\end{array}$ & Search for genes sul 1 & 417 & $\begin{array}{l}\text { Hao-Chang et al. } \\
\qquad(2012)\end{array}$ \\
\hline$q n r(\mathrm{~A})$ & $\begin{array}{l}\text { F: ATTTCTCACGCCAGGATTTG } \\
\text { R: GATCGGCAAAGGTTAGGTCA }\end{array}$ & \multirow[t]{3}{*}{ Search for $q n r$ genes } & 516 & \multirow{3}{*}{$\begin{array}{l}\text { Robicsek et al. } \\
\qquad(2006)\end{array}$} \\
\hline$q n r(\mathrm{~B})$ & $\begin{array}{l}\text { F: GATCGTGAAAGCCAGAAAGG } \\
\text { R: ACGATGCCTGGTAGTTGTCC }\end{array}$ & & 469 & \\
\hline$q n r(\mathbf{S})$ & $\begin{array}{l}\text { F: ACGACATTCGTCAACTGCAA } \\
\text { R: AAATTGGCACCCTGTAGGC }\end{array}$ & & 417 & \\
\hline
\end{tabular}


Table.2 ATB resistance profile of multidrug-resistant Salmonella serotypes (MDR) isolated from raw chicken gizzards

\begin{tabular}{|c|l|c|}
\hline Serotypes & \multicolumn{1}{|c|}{ Multidrug-resistant Serotype Profiles } & MRS \\
\hline Agona & TicTeSXT / TicCSXT & 4 \\
\hline Derby & TicCSXT / TicCTeSXT & 2 \\
\hline Hadar & TicSXTNalCipTe & 1 \\
\hline Budapest & TicTeSXT / TicCSXT / TiCTeSXTCTeSXT & 5 \\
\hline Riuru & CTeSXTNal / CTeSXT & 2 \\
\hline Essen & TicCTeSXT / AAMCTicSXTTe / SXTNalTe / TicGTeSXT & 4 \\
\hline Kentucky & GSXTNalCipTe / TicGTeSXT & 4 \\
\hline Chester & TicSXTTe & 1 \\
\hline
\end{tabular}

MRS: multiresistant strains; A: Amoxicillin; AMC: Amoxicillin / Clavulanic acid, Tic: Tircacillin; C:

Chloramphenicol; G: Gentamycin; Nal: Nalidixic acid; Cip: Ciprofloxacin; SXT: Cotrimoxazole; Te: Tetracycline.

Table.3 Strains with bla genes similar to strains isolated

\begin{tabular}{|c|c|c|c|}
\hline Salmonella isolated & NCBI Strains & Gene type bla & $\%$ identity \\
\hline & Salmonella Schwarzengrund S782 & bla CTX-M-2 & \multirow[t]{10}{*}{$96 \%$} \\
\hline & Salmonella Typhimurium 18-425 -M-5 & bla CTX-M-5 (ISEcp1) & \\
\hline & Escherichia coli BR-79 & bla CTX-M-2 (ISCR1) & \\
\hline & Escherichia coli KUN-9085 & bla CTX-M-44 & \\
\hline & Escherichia coli B275 & bla CTX-M-97 & \\
\hline \multirow[t]{6}{*}{ Salmonella Kentucky } & Escherichia coli E39 (ESBL) & bla CTX-M-92 & \\
\hline & Proteus mirabilis TUM11514 & bla CTX-M-2 & \\
\hline & Pseudomonas aeruginosa PHB 53 & blacTX-M-2 & \\
\hline & Klebsiella pneumoniae K6P & bla CTX-M-2 & \\
\hline & Klebsiella pneumoniae HB 99 & bla CTXM-59 & \\
\hline & Klebsiella oxytoca $76 \mathrm{C}$ & bla $\mathrm{OXY}$ & $100 \%$ \\
\hline Salmonella 0: 3.10 & Klebsiella pneumoniae & bla NDM-1 & $100 \%$ \\
\hline
\end{tabular}


Fig.1 Electrophoretic profile of the PCR amplification products of the sul 1 (A), tet (A) (B) and blaCTX-M (1) genes, existing in Salmonella strains isolated from raw chicken gizzards.
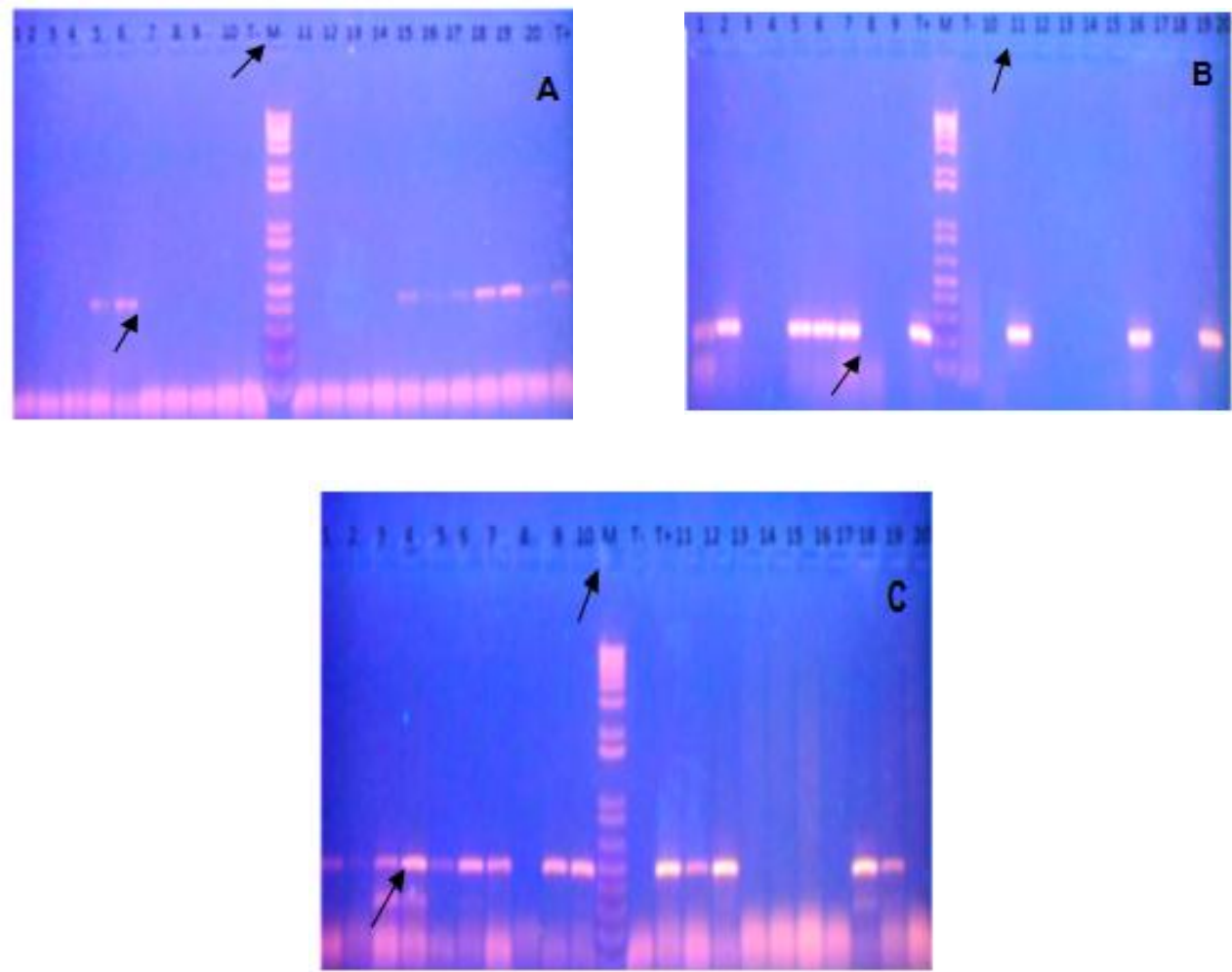

A: M. $1 \mathrm{~kb}(+)$ molecular weight marker (Eurogentec, Smart Ladder); T (-). The negative control. T (+). The positive control (E. coli DJ21-15). The amplicons positive for the sul1 gene have the expected size of $417 \mathrm{bp}$; B: M. $1 \mathrm{~kb}(+)$ molecular weight marker (Eurogentec, Smart Ladder); T (-). The negative control. T (+). The positive control (E. coli PSL 18X61367). The positive amplicons tet (A) gene have the expected size of 210 bp. tet (A): gene involved in resistance to tetracycline; C: M. $1 \mathrm{~kb}(+)$ molecular weight marker (Eurogentec, Smart Ladder); T (-). The negative control. T (+). The positive control (E. coliX92506). Amplicons positive for the bla CTX-M gene (1) have the expected size of $499 \mathrm{bp}$.

Overall, the same problems of resistance to antibiotics are found in strains of Salmonella whether they are of animal or human origin. Thus, the Salmonella strains isolated from poultry farm products are also affected by multidrug resistance to antibiotics. The direct involvement of $\beta$-lactams, sulfonamides, cyclins and fluoroquinolones, as well as the presence of resistance genes in our multidrugresistant strains could reflect their ability to develop resistance mechanisms, both genetic and biochemical, for the simple purpose of counterbalance their action.

Indeed, the bla CTX-M genes are those which mainly confer resistance to third generation cephalosporins such as cefotaxime (Arlet et al., 2006; Hur et al., 2010). The sequencing carried out on all of the amplicons of the degenerate primer bla CTX-M consensus revealed similarities varying from 96 to $100 \%$ with the bla sequences of bacterial strains contained in the NCBI database. These enzyme sequences are of bla CTX-M-2, -5, 44, -59, -92, -97, -131, bla NDM-1 and bla OXY type. These observations reflect the probable existence of a diversity of bla genes in the isolated Salmonella strains. The different types of bla genes obtained, belonging to classes $\mathrm{A}$ and $\mathrm{B}$ according to the classification of Ambler (1980), reflect the ability of our Salmonella strains to resist 
antibiotics of the $\beta$-lactam family, through the mechanism of enzymatic hydrolysis (enzymatic inactivation). The presence of mobile genetic elements, in particular the insertion sequences of the bla CTX-M 44 ISEcp1 and bla CTX-M-2 ISCR1 type, reflect the possible mobilization of the bla CTX-M genes. Indeed, the mobilization of bla CTXM genes is demonstrated experimentally by IS insertion sequences located upstream of the genes, such as ISEcp1 (Latirgue et al., 2006). In addition, the effect of the promoter of these insertion sequences, increasing the expression of bla CTX-M genes, suggests that these insertion sequences located upstream of these genes would play a role in the selection and dissemination of genes. bla CTX-M. Furthermore, this insertion sequence (ISEcp1) is found associated with the expression of all the groups of $\beta$-lactamases of the cefotaximase type with the exception of the bla CTX-M-8 group. The ISCR1 insertion sequence, for its part, is linked to several members of the CTXM-2 group (Barlow et al., 2008).

The presence of the tet (A) gene in multiresistant Salmonella strains possibly testifies to the involvement of the tet genes, in this case the tet (A) gene, in the resistance of Salmonella to tetracyclines. Indeed, several authors have put forward the hypothesis according to which bacteria have the capacity to develop a mechanism of resistance to this molecule (tetracycline), thanks to the existence of an efflux pump encoded by the genes tet, which would have the direct consequence of reducing the level of toxicity of this antibiotic within the bacteria (Butaye $e t$ al., 2003; Chopra and Roberts, 2001). These genes are also the most prevalent genetic determinants among Gram-negative bacteria including Salmonella (Schnabel and Jones, 1999; Carattoli et al., 2001). Resistance to sulfonamides generally arises according to Hendi et al., (2013), the acquisition of sul genes (sul1 and / or sul2) in Gram-negative bacilli. As a result, the acquisition of this resistance marker may reflect the ability of isolated strains to develop a resistance mechanism to sulfonamides by production of enzymes of the dehydropteroate synthetase type, encoded by the sul genes (Huovinen et al., 1995).

Regarding bacterial resistance to fluoroquinolones, phenotypic tests revealed a considerable rate of resistance to ciprofloxacin $(30.77 \%)$. However, molecular tests did not reveal the presence of any of the $q n r$ genes tested for resistance to ciprofloxacin by PCR.

This absence can be explained by the fact that in addition to the $q n r$ genes, resistance to fluoroquinolones can also be the plasmid factor such as the variant gene aac6'-Ibcr, which increases the level of resistance of Salmonella strains to fluoroquinolones. Indeed, from 2009 to 2011, antibiotic sensitivity studies carried out by the National Salmonella Research Center in Paris, on strains of various contamination origins made it possible to confirm the presence of these two types of resistance markers involved in resistance to fluoroquinolones for the serotypes Agona (India), Nima (Mali) and Typhimurium (Unknown) (Weill and Le Hello, 2011). Thus, the resistance to fluoroquinolones of the isolated Salmonella strains can be attributed to the aac6'-Ibcr gene.

This work showed the presence of strains of Salmonella highly resistant to antibiotics in the raw gizzards of chickens analyzed. These multiresistant Salmonella strains, carriers of plasmid support resistance genes, give them the power to disseminate in the environment. This could constitute a real risk for the health of the consumer.

The monitoring of the evolution of antibiotic resistance in the Ivorian poultry sector 
therefore remains the sine qua non to overcome the appearance of such strains in order to guarantee the safety of food in general and those based on food. poultry in particular.

\section{References}

Ambler, R P., 1980. The structure of betalactamases. Phylosophycal Transactions of the Royal Society of London (Biol.). 289, 321-331.

Anonymous 1. 2006. The community summary report on trends and sources of zoonoses, zoonotic agents. Antimicrobial resistance and foodborne out breaks in the European Union in 2005. European Food Safety Authoriry. 94, 234.

Anonymous 2. 2002. Risk assessment of Salmonella in eggs and broilers. WHO /FAO. Microbiological Risk Assessment Series 1. Interpretative summary. $77 \mathrm{p}$.

Anonymous 3. 2009. Federal Office of Public Health 1999. Antibiotic resistance of bacteria in the field of human, veterinary and food medicine. Information service report. Bern. 30p.

Arlet, G., Barret, T J., Buttaye, P., Cloeckaert, A., Mulvey, M R., and White, D G. 2006. Salmonella resistant to extended-spectrum Cephalosporins: Prevalence and epidemiology. Microbes and Infection. 8, 1945-1954.

Barlow, M., Reick, A., Jacobs, S D., Medina, M., Meyer, M P J., McGowan, Jr. E., and Tenover, FC. 2008. High rate of mobilization for bla CTX-Ms. Emerging Infection Diseases. 14, 423428.

Bertrand, S., Baeyens, D., De Cooman, F., Steenhaut, H., Dupon, G., and Thirionet, M. 2010. Surveillance data from the National Reference Center for Salmonella and Shigella, Belgium 2010. Activity report 2010. 44 p.
Bourgeois, N., Savard, B., and Moubareck, C. 2003. Interspecies transfer of vancomycin resistance from poultry Enterococcus faecium to human Enterococcus faecal is in digestive tract of human flora associated mice. In Abstract of the Forty-third. Interscience Conference on Antimicrobial Agents and Chemotherapy, Chicago. Pp. 83-84.

Butaye, P., Cloeckaert, A., and Schwarz, S. 2003. Mobile genes coding for effluxmediated antimicrobial resistance in Gram-positive and Gram-negative bacteria. International Journal of Antimicrobial Agents. 22, 205-210.

Carattoli, A., Villa, L., Pezzella, C., Bordi, E., and Visca, P. 2001. Expanding Drug Resistance through Integron Acquisition by IncFI Plasmids of Salmonella enterica Typhimurium, Emerging Infectious Diseases. 7, 444447.

Chaiba, A., Rhazi, FF., Chahlaoui, A., Soulaymani, B R., and Zerhouni, M. (2008). Occurrence of Salmonella in Chicken Carcasses and Giblets in Meknès Morocco. Pakistan Journal of Nutrition. 7, 231-233.

Chopra, I., and Roberts, M. 2001. Tetracycline antibiotics: mode of action, applications, molecular biology and epidemiology of bacterial resistance. Microbiology and Molecular Biology Reviews. 65, 232-260.

CLSI. 2005. Clinical Laboratory Standard Institute. Performance Standards for Antimicrobial Susceptibility Testing (15th information supplement). CaLS Institute Ed: Wayne, Pennsylvania.

Fablet, C., Beloeil, P.-A., Fravalo, P., Jolly, J.P., Eveno, E., Hascoet, Y., Salvat, G., and Madec, F. 2003. Study of circumstances associated with the shedding of Salmonella enterica by growing pigs. Pig Research Days. 35, 
401-408.

Gaedirelwe, O G., and Sebunya, T K. 2008. The prevalence and antibiotic susceptibility of Salmonella $\mathrm{sp}$ in poultry and ostrich samples from slaughter houses in Gaborone; Botswana. Journal of Animal and Veterinary Advances. 7, 1151-1154.

Hao-Chang, S., Guang-Guo, Y., Ran, T., RuiQuan, Z., Jian-Liang, Z. and YouSheng, L. 2012. Class 1 and 2 integrons, sul resistance genes and antibiotic resistance in Escherichia coli isolated from Dongjiang River, South China. Environmental Pollution. 169, 42-49.

Hendi, A K., Shubbar, E E., and Addos, S A. 2013. Molecular study on distribution of sul 1 and sul 2 genes among Salmonella enterica causing enteric fever in magazine of Al Kufa University for Biology. 5, 52-58.

Huovinen, P., Sundstrom, L., Swedberg G., and Skold, O. 1995. Trimethoprim and sulfonamide resistance. Antimicrobial Agents and Chemotherapy. 39, 279289.

Hur, J., Kim, J H., Park, J H., Lee, Y J., and Lee, J H. 2010. Molecular and virulence characteristics of multidrug resistant Salmonella Enteritidis strains isolated from poultry. The Veterinary Journal. 189, 306-311.

ISO-6579. 2002. Food microbiology Horizontal method for the detection of Salmonella spp. V08-013 2002. Pp 139.

Jianhua, G., Robert, J F., Hai, Y., Chambers, J R., Wheatcroft, R., Sabour, P M., and Shu, C. 2002. Molecular analysis of bacterial populations in the ileum of broiler chickens and comparison with bacteria in the cecum. FEMS. Microbiology and Ecology. 41, 171179.

Karou, G T., Bonny, A C., Ouattara, G H.,
Dadie, A T., and Ahonzo-Niamke, S L. 2013. Prevalence of Salmonella and antimicrobial resistance of serovars in retail chicken gizzards. International Journal of medical and applied Sciences. 2, 2320-3137.

Karraouan, B., Fassouane A., El Ossmani, H., Cohen, N., Charafeddine, O., and Bouchrif, B. 2010. Prevalence and virulence genes of Salmonella isolated from raw ground turkey meat in Casablanca (Morocco). Journal of Veterinary Medicine. 161, 127-132.

Kauffmann, F., and White, B P. 1934. Enterobacteriacea. 2nd. ed. E. Munksguard, Copenhagen. Salmonella. Commettee. Savage, WG and. P. Bruce White. 1945. An investigation of the Salmonella group, with special reference to food poisoning, Journal of Hygiene. 118, 368-384.

Kiiru, J., Kariuki, S., MGoddeeris, B., and Butaye, P. 2012. Analysis of $\beta$ lactamase phenotypes and carriage of selected $\beta$-lactamase genes among strains obtained from Kenyan patients during an 18-year period. BMC Microbiology. 12, 155.

Lartigue, M F., Poirel, L., Aubert, D., and Nordmann, P. 2006. In vitro analysis of ISEcp1B mediated mobilization of naturally occuring $\beta$-lactamase gene blaCTX-M of Kluyvera ascorbata. Antimicrobial Agents and Chemotherapy. 50, 1282-1286.

Moubareck, C., Bourgeois, N., and Courvalin, P. 2003. Multiple antibiotic resistance gene transfert from animal to human enteroccoci in the digestive tract of gotobiotic mice. Antimicrobial Agents and Chemotherapy. 47, 2993-2996.

Ng, L.-K., Martin, I., Alfo, M., and Mulvey, M. 2001. Multiplex PCR for the detection of tetracycline resistance genes. Molecular Cellular Probes. 15, 
209-215.

Ouattara, N D., Guessend, N., Gbonon, V., Toe, E., Dadié, T., and Tiécoura, B. 2013. Consumption of Antibiotics in the Avian Sector in Abidjan: Case of some semi-industrial farms. European Journal of Scientific Research. 94, 8085.

Prin, S., Bastianelli, D., and Saboulard, M. 2001. Poultry production in the world: A strong dynamic. Agroligne $\mathrm{n}{ }^{\circ} 18$. November- December 2001. 11-13.

Robicsek, A., Strahilevitz, J., Sahm, DF., Jacoby, GA., and Hooper, DC. 2006. $q n r$ Prevalence in CeftazidimeResistant Enterobacteriaceae Isolates from the United States. Antimicrobial Agents and Chemotherapy. 50, 28722874.

Rozilla, A., Nur-Harni, A., Tan, D., and Wee, S. 2007. Rapid molecular detection of Salmonella isolated from poultry farm. 19 th Veterinary Association Congress, Malaysia. 201-203.

Schnabel, E., and Jones, A. 1999. Distribution of Tetracycline Resistance Genes and Transposons among Phylloplane Bacteria in Michigan Apple Orchards. Applied Environment and Microbiology. 65, 4898-4907.

Tao, Y., Xiaojie, J., Qiaohong, Z., Junmei, W., and Zhenbin, W. 2014. Antimicrobial resistance, class 1 integrons, and horizontal transfer in Salmonella isolated from retail food in Henan, China. Journal of Infectious Deseases in Development Countries. 8, 705-711.

Traoré, I. 2003. Portage and antibiotic resistance of Salmonella strains isolated from the viscera of chickens sold in the markets of Abobo (Abidjan), Côte d'Ivoire, at the Food Science and Technology UFR, University of Abobo-Adjamé : Abidjan, Ivory Coast. 58p.

Turki, Y., Ouzari, H., Mehri, I., Ben Aissa, R., and Hassen, A. 2011. Biofilm formation, virulence and multidrug resistance in Salmonella Kentuky. Food Rechearch International. 45, 940946.

Ungemach, F R., Müller-Bahrdt, D., and Abraham, G. 2006. Guide lines for prudent use of antimicrobials and their implications on antibiotic use in veterinary medicine. International Journal of Medical Microbiology. 296, 33-38.

Weill, F X., and Le Hello, S. 2011. National Reference Center for Salmonella. 2011 annual activity report. Enteric pathogenic bacteria research and expertise unit. Institut Pasteur, Paris (France). 72p.

\section{How to cite this article:}

Bonny Aya Carole and Karou Tago Germain. 2021. Phenotypic and Molecular Characterization of Antibiotic resistance of Isolated Salmonella Strains from Chickens in Côte D'ivoire. Int.J.Curr.Microbiol.App.Sci. 10(07): 48-58. doi: https://doi.org/10.20546/ijcmas.2021.1007.006 